\title{
Amine Molecular Cages as Supramolecular Fluorescent Explosive Sensors: A Computational Perspective
}

\author{
Martijn A. Zwijnenburg, ${ }^{* \dagger}$ Enrico Berardo, ${ }^{\ddagger}$ William J. Peveler, ${ }^{\dagger}$ and Kim E. Jelfs ${ }^{\dagger}$ \\ ${ }^{\dagger}$ Department of Chemistry, University College London, 20 Gordon Street, London WC1H 0AJ, U.K. \\ ${ }^{\ddagger}$ Department of Chemistry, Imperial College London, South Kensington, London SW7 2AZ, U.K.
}

\section{Supporting Information}

ABSTRACT: We investigate using a computational approach the physical and chemical processes underlying the application of organic (macro)molecules as fluorescence quenching sensors for explosives sensing. We concentrate on the use of amine molecular cages to sense nitroaromatic analytes, such as picric acid and 2,4dinitrophenol, through fluorescence quenching. Our observations for this model system hold for many related systems. We consider the different possible mechanisms of fluorescence quenching: Förster resonance energy transfer, Dexter energy transfer and photoinduced electron transfer, and show that in the case of our model system, the fluorescence quenching is driven by the latter and involves stable supramolecular sensor-analyte host-guest complexes. Furthermore,

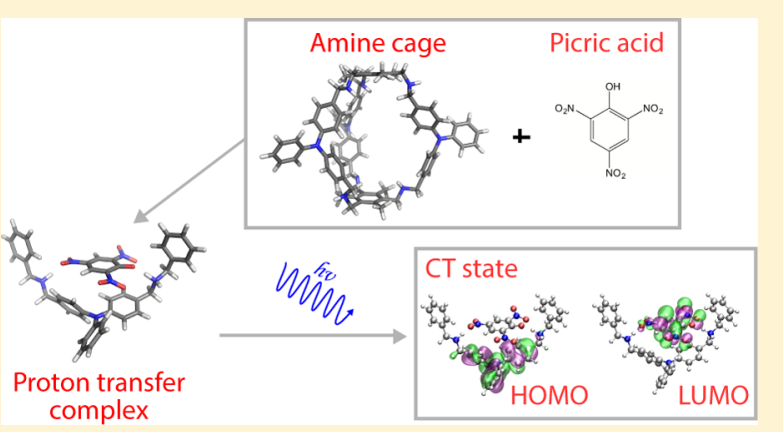
we demonstrate that the experimentally observed selectivity of amine molecular cages for different explosives can be explained by the stability of these host-guest complexes and discuss how this is related to the geometry of the binding site in the sensor. Finally, we discuss what our observations mean for explosive sensing by fluorescence quenching in general and how this can help in future rational design of new supramolecular detection systems.

\section{INTRODUCTION}

The quenching of a material's fluorescence by the constituents of explosives, such as picric acid (PA) or trinitrotoluene (TNT), provides a cheap and convenient way of detecting explosives (see Scheme 1), as well as sensing their

Scheme 1. Structures of Picric Acid (A), Trinitrotoluene (B), 2,4-Dinitrophenol (C), and Nitrobenzene (D)<smiles>O=[N+]([O-])c1cc([N+](=O)[O-])c(O)c([N+](=O)[O-])c1</smiles>

A

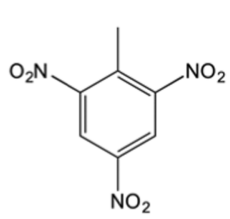

B

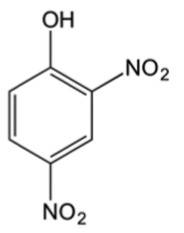

$\mathrm{C}$

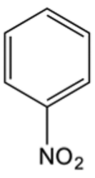

$\mathrm{D}$ decomposition products in the environment. This approach to explosive detection, inspired by earlier work on the sensing of gases, small ions and biomolecules through fluorescence quenching, has the potential to yield sensors with high sensitivity and specificity through control of a fluorescent material's optical properties and the supramolecular interaction between that material and the target explosive. Fuelled in part by the uncertain times we live in, there is consequently a large research activity focused upon the development of fluorescent polymers, $^{1-4}$ metal organic frameworks, ${ }^{5-12}$ metal organic cages, ${ }^{13}$ nanoparticles, ${ }^{14-17}$ and (small) organic molecules ${ }^{18,19}$ for explosive sensing.

One such class of new systems for explosive detection through fluorescence quenching are amine molecular cages, $^{20-23}$ synthesized through dynamic covalent imine chemistry $^{24}$ followed by imine reduction. Beyond sensing explosives $^{25,26}$ and other chemicals, ${ }^{27}$ amine molecular cages show promise for application in catalysis ${ }^{28}$ and separation, ${ }^{29-32}$ because of the presence of an intrinsic internal cavity, as well as the fact that they can be easily modified by synthetic chemistry, and are soluble in common solvents and hence are easily processable.

Mukherjee and Acharyya showed that one amine molecular cage containing both secondary amine and strongly fluorescent triphenylamine groups (see Figure 1A) had a strong specificity for PA over other nitroaromatic molecules, ${ }^{25}$ where PA quenched the cage fluorescence 5 times more strongly than the next most potent fluorescence quenching explosive: 2,4dinitrophenol (24DNP, see Scheme 1C). They also found that converting the secondary amine groups in the cage molecule into tertiary amines, via a copper(I) catalyzed three-component coupling reaction, results in the modified cage being less specific for $\mathrm{PA}^{26}$ The fluorescence quenching efficiency for $24 \mathrm{DNP}$ in the case of this so-called "decorated cage" is almost

Received: March 24, 2016

Revised: $\quad$ May 5, 2016

Published: May 5, 2016 


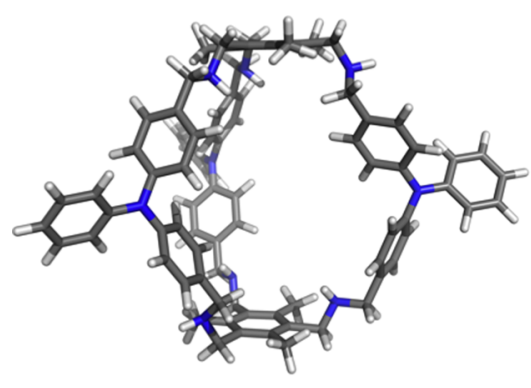

A

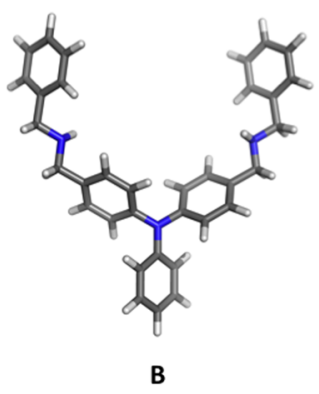

B
Figure 1. B3LYP/DZP-optimized 3D structures of the amine cage (A) and cluster model 1 (B).

$90 \%$ that of PA. In this paper, we focus on these amine cage molecules as a model system to explore, from a computational and theoretical point of view, the (photophysical) origin of sensitivity and specificity in fluorescence quenching sensing. We hope the insight obtained can help in the future rational development of supramolecular fluorescent explosive sensors.

Quenching of the fluorescence of a sensor molecule after the introduction of an analyte can either be the result of (i) energy transfer or (ii) electron transfer between the sensor and analyte. In the case of energy transfer, a real or virtual photon (Förster resonance energy transfer, FRET, or Dexter energy transfer, DET) is exchanged between the sensor and the analyte, resulting in the transfer of the excited state from the former to the latter (see Figure 2). Alternatively, an electron might be

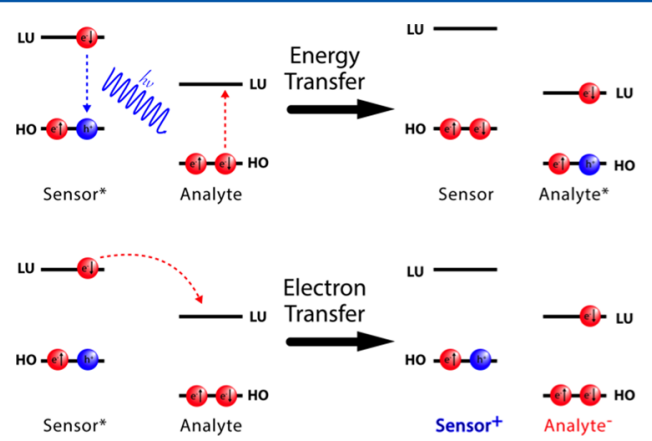

Figure 2. Illustration of fluorescence quenching through energy transfer (top) and electron transfer (bottom). Electrons in red and holes in blue.

transferred through photoinduced electron transfer (PET), where the excited state of the sensor effectively reduces the analyte (see Figure 2). In both scenarios fluorescence is quenched, or at least shifted to a longer wavelength than the original sensor fluorescence. In the case of FRET/DET this quenching is due to fact that the excited analyte fluoresces at much longer wavelength or does not fluoresce at all. In the case of PET, in contrast, the excited state has been converted into a charge-separated state, with an excess electron on the analyte and a hole on the sensor, which cannot decay via the emission of light without significant thermal activation.

One can distinguish between dynamic and static quenching, where the fluorescence lifetime of the sensor is reduced after addition of the analyte in dynamic quenching and does not change in the case of static quenching. On the microscopic level, the difference between both scenarios is thought to be related to the question of whether the sensor and analyte form a stable complex in the ground state (static quenching) or do not and thus only collide, or more generally come close enough for interaction, after the sensor has already been excited (dynamic quenching). Experimentally it has been suggested, ${ }^{25}$ based on fluorescence lifetime measurements, that the quenching in the case of the amine cages is, at least for PA, static rather than dynamic in nature, suggesting a strong interaction between sensor and analyte.

Here we use a combination of (time-dependent) density functional theory ((TD)-DFT) and approximate couple cluster theory (RI-CC2) calculations to shed light on the nature of the exact microscopic quenching mechanism in these cages, the reason why quenching is static and why the degree of fluorescence quenching is much more pronounced for PA than other analytes such as $24 \mathrm{DNP}$, nitrobenzene (NB) and TNT in the case of the cage with secondary amine groups. We will also discuss why the decorated cage is less specific for PA and more sensitive to other explosives. Finally, we will discuss what one can learn about supramolecular explosive sensing from these results and the potential role for computationally guided rational design of such materials.

\section{METHODOLOGY}

Modeling the molecules and host-guest assemblies that underlie sensing by fluorescence quenching is a significant challenge. One needs to employ a theoretical method that can sufficiently describe the weak dispersive interactions between the cage host and analyte guest as well as the optical properties of such assemblies and their constituent parts. The computational method must also be sufficiently computationally cheap to allow for calculations on systems with tens to hundreds of atoms. In practice, such a method does not currently exist and the calculations in this paper instead use a combination of methods. Ground state geometries are obtained using density functional theory (DFT) calculations with the B3LYP 33,34 hybrid density functional and its dispersion corrected version $\mathrm{B} 3 \mathrm{LYP}+\mathrm{D} 3{ }^{35}$ which includes an empirical correction that improves the description of long-range attractive dispersion forces. Vertical excitation calculations and excited state optimizations for predictions of fluorescence energies are obtained by TD-DFT calculations using the B3LYP or CAMB3LYP ${ }^{36}$ density functionals. CAM-B3LYP is an example of a range-separated functional, where the percentage of HartreeFock exchange included when calculating the exchangecorrelation energy for a certain volume element depends upon the interelectronic separation. Use of this functional has the advantage over calculations based on simpler hybrid functionals, such as B3LYP, in that charge-transfer (CT-) excitations are better described, as discussed in more detail below. This improved description of CT-states comes at the expense of an overall upward (blue) shift of the predicted excitation spectrum and the absence of parameters for the empirical dispersion correction in the literature for CAMB3LYP. Finally, we performed vertical excitation calculations and even excited state relaxations using the approximate single and doubles coupled cluster method RI-CC2. ${ }^{37,38}$ RI-CC2 is inherently more robust and accurate than TD-DFT but also computationally much more expensive, making RI-CC2 calculations tractable only as a benchmark for selected structures.

As touched upon above, the description of charge-transfer states can be problematic in TD-DFT. Specifically, the excitation energies when there is negligible overlap between the occupied and unoccupied orbitals involved will be severely 
underestimated by nonhybrid or hybrid functionals with a small percentage of Hartree-Fock exchange (e.g., B3LYP). A diagnostic for such problematic CT-excitations is the $\Lambda$ diagnostic by Peach et al., ${ }^{39}$ which for a given excitation takes values in the range from 0 , no overlap between the occupied and unoccupied orbitals involved in the excitation, to 1 , complete overlap of the orbitals. Previous work by Peach et al. ${ }^{39}$ suggests that TD-B3LYP excitations with a $\Lambda$ value of less than 0.3 should be treated with care. They also found that for a density functional such as CAM-B3LYP, there was no correlation between the $\Lambda$ value and the quality of the TDDFT description of excitations. Indeed, CAM-B3LYP should not suffer from problems describing CT-states due to the large percentage of Hartree-Fock exchange included at large interelectronic separations.

All B3LYP $(+\mathrm{D} 3)$ calculations, except those used to extract $\Lambda$ diagnostic values, and all RI-CC2 calculations, were performed using Turbomole $6.6 .^{40,41}$ All CAM-B3LYP calculations, except a selected number exploiting symmetry, and B3LYP calculations for $\Lambda$ diagnostic values were performed using GAMESS$\mathrm{US}^{42}$ (version 1 May 2013 (R1)). The CAM-B3LYP calculations with symmetry were performed using NWChem ${ }^{43}$ 6.5. Unless otherwise stated, the B3LYP calculations used the $\mathrm{DZP}^{44}$ basis-set, the CAM-B3LYP calculations the 6$31 \mathrm{G}^{* * 45,46}$ basis-set and the RI-CC2 calculations either a smaller def2-SVP ${ }^{47}$ or larger def2-TZVPP ${ }^{47}$ basis-set.

Some of the (TD-)DFT calculations were performed in the presence of a dielectric screening model to include bulk solvent effects. The relevant (TD-)B3YLP Turbomole calculations used the COSMO solvation model, ${ }^{48}$ with all reported COSMO energies including the outlying charge correction, while the (TD-)CAM-B3LYP calculations in GAMESS-US that include solvation used a combination of the $\mathrm{PCM}^{49,50}$ and $\mathrm{SMD}^{51}$ models. In both cases the solvent was dichloromethane (DCM), modeled using a relative dielectric permittivity $\varepsilon_{r}$ value of 9 in the case of COSMO and 8.93 in the case of $\mathrm{PCM} / \mathrm{SMD}$.

Estimates of the vibrational, rotational and translational contributions to binding free energies, were based on the result of numerical frequency calculations on the relevant structures. No scaling was applied to the DFT frequencies but besides the conventional approximation (below referred to as the harmonic approximation), in which the contribution of every vibrational mode to the vibrational entropy is described as a harmonic oscillator, we also explored the approximation by Grimme ${ }^{52}$ (below referred to as the harmonic + rotor approximation) in which the contribution of low frequency modes $\left(<100 \mathrm{~cm}^{-1}\right)$ was modeled as arising from free rotors rather than harmonic oscillators (using $\omega_{0}=100 \mathrm{~cm}^{-1}$ and $\alpha=4$ ). The latter approximation corrects for the fact that the standard harmonic approximation overestimates the contribution to the vibrational entropy of low frequency modes. The nonelectronic part of the free energies in the harmonic + rotor case was obtained by subtracting $\left(-T^{*} S_{\text {vib,harmonic }}\right)$ and adding $\left(-T^{*} S_{\text {vib,harmonictrotor }}\right)$ to the value reported by the "freeh" module of Turbomole. Finally, for reasons of computational tractability, in all cases the nonelectronic parts of the free energies reported were calculated by frequency calculations using the DZP basis-set on B3YLP/DZP optimized geometries, even when the electronic energies were calculated using def2-TZVP.

\section{RESULTS AND DISCUSSION}

We will now attempt to answer the questions about the nature and mechanism of fluorescence quenching outlined at the end of the introduction. However, before doing so we will first explore our ability to predict the optical properties of the isolated cage.

Optical Properties of the Isolated Cage. Optical Absorption Spectrum of the Isolated Cage. The optical absorption spectrum of the isolated amine cage was calculated using both TD-DFT and RI-CC2 for the whole cage and also a cluster model (1) comprising of one of the three edges of the cage (see Figure 1B and Scheme 2). In the former case, we

\section{Scheme 2. Structure of Cluster Model 1}

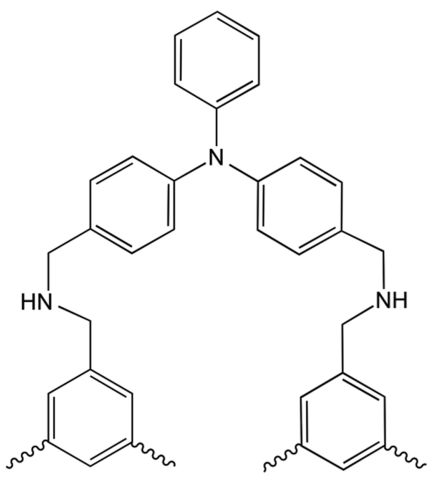

considered (i) the conformation obtained from the solvate single-crystal X-ray diffraction structure (CCDC number $1018380, C_{2}$ symmetry), ${ }^{25}$ (ii) the expected conformation in solution obtained by using the inflation approach of Santolini et al., ${ }^{53}$ which models the effect of solvation on the cage conformation in a mean field manner through a spherical constraint on the cavity, and (iii) the lowest energy collapsed conformer found in a conformer search ${ }^{53}$ (see Figure S1 in the Supporting Information for the 3D B3LYP/DZP optimized structures of the different conformers).

Concentrating first on the whole cage, Table 1 gives the lowest vertical TD-B3LYP excitation energies, referred to below as the optical gap, for the different models, where we also

Table 1. TD-B3LYP/DZP Predicted Lowest Vertical Excitation Energy of the Amine Cage for a Structure Obtained When Starting the B3LYP/DZP Ground State Geometry Optimization from (i) the Experimental Conformation of the Solvated Cage (XRD), (ii) the Conformer Obtained Using the Approach of Santolini and Co-Workers (Inflated), and (iii) the Lowest Energy Conformer of the Cage (Collapsed) ${ }^{a}$

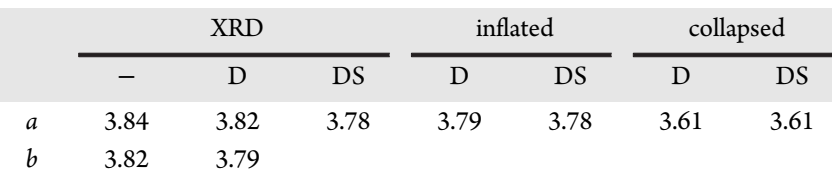

${ }^{a}$ For all three structures we also considered the effect of using Grimme's DFT+D3 dispersion correction to DFT (D) and a combination of the dispersion correction and the COSMO dielectric screening solvation model $\left(\varepsilon_{\mathrm{r}} 9\right.$, DCM, DS). For the XRD structure with $C_{2}$ symmetry, the lowest vertical excitation energies belonging to either of the two irreducible representations are shown. All values are in $\mathrm{eV}$. 
explore the effect of solvent and dispersion. It is apparent that there are only very small differences in the predicted optical gap when starting the preceding ground state optimization from either the conformation of the solvate crystal structure or the solution conformation predicted by Santolini et al. ${ }^{53}$ The collapsed structure, corresponding to the lowest energy conformation predicted by conventional conformer searching algorithms, however, has a red-shifted optical gap. The addition of a dispersion correction, which corrects for the absence of a proper description of long-range dispersive interaction in DFT, and a dielectric solvent model to represent bulk solvation, only leads to minor changes in the optical gap in all cases. Finally, ignoring the collapsed conformation, which is unlikely to exist in the DCM solution used experimentally for the fluorescence quenching studies, there is a decent match between the optical gap of the amine cage and the experimental absorption onset of the amine cage in solution (longest wavelength peak maximum at $\left.\sim 310 \mathrm{~nm},{ }^{25} 4 \mathrm{eV}\right)$.

Moving our focus to the cluster model 1, Tables 2 and S1 show that the TD-B3LYP predicted lowest vertical excitation

Table 2. Predicted Lowest Vertical Excitation Energies Belonging to the $a$ and $b$ Irreducible Representations of the Mukherjee Amine Cage Cluster Model $1^{a}$

\begin{tabular}{|ccccc} 
& TD-B3LYP & RI-CC2/SV & RI-CC2/TZ & TD-CAM-B3LYP \\
\hline$a$ & $4.07 / 3.99$ & $4.40(4.29)$ & 4.18 & $4.60(4.64)$ \\
$b$ & $3.83 / 3.69$ & $4.18(4.06)$ & 3.86 & $4.52(4.52)$
\end{tabular}

${ }^{a_{T}}$ The structure is optimized in the $\mathrm{C}_{2}$ point group using B3LYP/DZP, whereas the vertical excitations are calculated using TD-B3LYP/DZP, RI-CC2/def2-SVP, RI-CC2/def2-TZVPP and TD-CAM-B3LYP/6$31 \mathrm{G}^{* *}$ respectively. In the case of RI-CC2/def2-SVP and TD-CAMB3LYP, results for calculations on a RI-CC2/def2-SVP and CAMB3LYP/6-31G** optimized geometry respectively are given in parentheses, while for the case of TD-B3LYP results obtained with the def2-TZVP basis-set are shown behind the slash. All values are in $\mathrm{eV}$.

energies agree well with the results of more accurate and more computationally expensive RI-CC2/def2-TZVPP calculations, both in terms of the absolute values and the ordering of excitations. The TD-B3LYP results for the cluster model $\mathbf{1}$ also agree very well with those for the whole cage in Table 1 , validating the utility of the cluster model. The TD-CAMB3LYP excitation energies, in contrast to the TD-B3LYP excitation energies, are, as to be expected from the literature, all blue-shifted to slightly higher energy $(0.5-0.7 \mathrm{eV})$, but again match the RI-CC2 results in terms of excitation ordering. Finally, the effect of the density functional used for the ground state optimization on the TD-CAM-B3LYP vertical excitation energies was found to be very small, while the effect of moving from DZP to the larger def2-TZVP basis-set is also found to be relatively small.

Fluorescence Spectrum of the Isolated Cage. Predictions of the fluorescence energy, where the energy of the lowest energy singlet ( 1$)$ is minimized and the fluorescence energy is obtained as the vertical difference between $S 1$ and the ground state $\mathrm{S} 0$ at this $\mathrm{S} 1$ minimum energy geometry (points $\mathrm{B}^{\prime}$ and $\mathrm{B}$ in Figure 3), are only computationally feasible for cluster model 1. As can be seen in Table 3, relaxation of S1 with TD-B3LYP yields a fluorescence energy that lies very close to experimental fluorescence maximum for the cage of $2.85 \mathrm{eV}(435 \mathrm{~nm})$. Calculation of Peach's $\Lambda$ diagnostic for S1 at this geometry (1), however, yields a value lower than 0.3 , strongly suggesting that

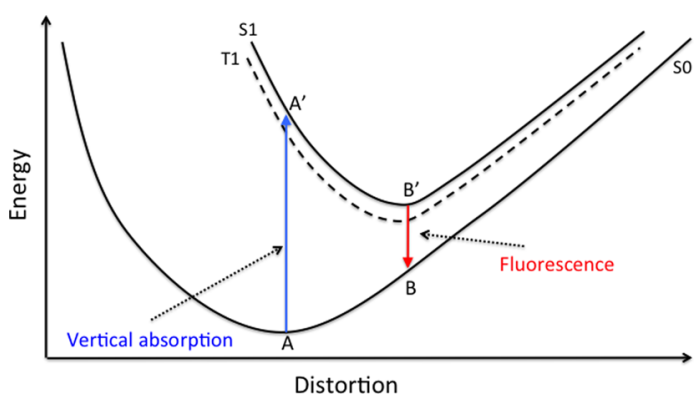

Figure 3. Schematic showing the absorption $\left(A \rightarrow A^{\prime}\right)$, excited state relaxation $\left(\mathrm{A}^{\prime} \rightarrow \mathrm{B}^{\prime}\right)$, and fluorescence $\left(\mathrm{B}^{\prime} \rightarrow \mathrm{B}\right)$ process occurring in a material.

this good match to experiment is accidental and that (1) is an artificial excited state minimum, which results from the spurious energetic stabilization of CT-states (see below). This analysis is further supported by the fact that a RI-CC2/def2-TZVPP single point calculation on the TD-B3LYP S1 minimum energy geometry predicts a lowest vertical excitation energy that is 0.6 $\mathrm{eV}$ larger than the TD-B3LYP fluorescence energy. Relaxation of S1 using TD-CAM-B3LYP, which by design does not suffer from the problem with describing CT-states, finds an alternative minimum (2), which indeed does not correspond to a CT-state. TD-B3LYP and RI-CC2/def2-TZVPP single point calculations on that geometry yield fluorescence energies that, at 3.18 and $3.27 \mathrm{eV}$, are slightly blue-shifted compared to experiment.

S1 optimizations using RI-CC2/def2-SVP rather than TDDFT yield two different stationary points. We focus in the remainder of this paper in terms of RI-CC2 obtained geometries on the lower energy stationary point (4). See section ESI-3 of the Supporting Information for a more indepth discussion of the RI-CC2 minima. TD-B3LYP and CC2/ def2-TZVPP single point calculations on this geometry yield fluorescence energies of 3.04 and $3.13 \mathrm{eV}$ respectively, both lying close to the experimental fluorescence maximum, as well as to the TD-B3LYP and CC2/def2-TZVPP single point values for the TD-CAM-B3LYP S1 minimum (2) $(3.27$ and $3.16 \mathrm{eV}$ respectively). Just as at the latter geometry, $\mathrm{S} 1$ at stationary point (4) is not a CT-state and use of TD-B3LYP is thus non problematic. Section ESI-4 of the Supporting Information discusses the energetic and structural changes associated with excited state relaxation, including the good structural fit between (2) and (4).

The lifetime predicted by the oscillator strength of the $S 1$ excited state is $72-98 \mathrm{~ns}$ at the ground state geometry, 50-100 ns at relaxed excited state geometry (2) and 79-172 ns at (4). The range of values predicted for each geometry results from the fact that in TD-DFT the oscillator strength and other properties depend on the gauge used to calculate them. These values are approximately ten times as large as the experimental lifetime $(\sim 10 \mathrm{~ns}),{ }^{25}$ suggesting perhaps a role of conical intersections in the experimental de-excitation.

The Potential of Dynamic Quenching and the Isolated Molecule Perspective. Energy Transfer. An overlap between the fluorescence spectrum of the cage and the absorption spectrum of the analyte is a key requirement for energy transfer and, thus, fluorescence quenching, via FRET/DET to be possible. To analyze the ability of the cage fluorescence to be quenched through FRET/DET we thus need to compare the predicted fluorescence signal for the cage from Table 3 with the 
Table 3. Predicted Fluorescence Energy of the Mukherjee Amine Cage Cluster Model $\mathbf{1}^{a}$

\begin{tabular}{|c|c|c|c|c|c|}
\hline & \multicolumn{2}{|c|}{ TD-B3LYP } & \multirow[b]{2}{*}{$\mathrm{RI}-\mathrm{CC} 2 / \mathrm{SV}$} & \multirow[b]{2}{*}{$\mathrm{RI}-\mathrm{CC} 2 / \mathrm{TZ}$} & \multirow[b]{2}{*}{ TD-CAM-B3LYP } \\
\hline & - & $S$ & & & \\
\hline (1) & $3.01(0.20)$ & - & 3.84 & 3.62 & 3.89 \\
\hline (2) & $3.18(0.66)$ & 3.16 & 3.49 & 3.27 & 3.68 \\
\hline (3) & $3.41(0.63)$ & - & 3.73 & 3.43 & 4.07 \\
\hline (4) & $3.04(0.63)$ & 3.03 & 3.34 & 3.13 & 3.56 \\
\hline
\end{tabular}

${ }^{a}$ Structure optimized without symmetry constraints using TD-B3LYP/DZP (1), TD-CAM-B3LYP/6-31G** (2), or RI-CC2/def2-SVP (3), and (4), followed by single point calculation using the alternative set-ups and RI-CC2/def2-TZVPP (all single point results in bold). In the case of TDB3LYP, the value of the $\Lambda$ diagnostic for the respective excitation (calculated using TD-B3LYP/6-31G**) is also given in between parentheses. The TD-B3LYP values in the column labelled $S$ were obtained using the COSMO dielectric screening solvation model $\left(\varepsilon_{\mathrm{r}} 9\right.$, DCM). All values are in eV.

optical gap of the analytes in Table S4. Such a comparison shows that only for the conjugate base anion of the "acid" analytes, e.g. the picrate anion in the case of picric acid, is there likely to be an overlap between the fluorescence spectrum of the cage and the absorption spectrum of the analyte. In the case of NB and undissociated 24DNP and PA, the predicted optical gap of the analytes is considerably larger than the predicted fluorescence energy of the cage cluster model and the (virtual) photons emitted by the latter are not energetic enough to excite the former. This all appears in line with what would be expected from the experimental spectra of both the cage and analytes. Dynamic fluorescence quenching through energy transfer, be it through FRET or DET, is thus predicted to only be viable for "acid" analytes such as PA and 24DNP and then only in the presence of a solvent or base that would (partially) deprotonate them and give rise to the conjugate base anion. As discussed in the Supporting Information (section 5), there is some suggestion that water (for example, as an impurity resulting from the fact that $\mathrm{PA}$ is typically stored wet) might be able to do this. ${ }^{54,55}$

Electron Transfer. For fluorescence quenching of the cage by the analytes through photoinduced electron transfer to be feasible, the excited state ionization potential ${ }^{56,57}$ of the cage (IP*, the potential associated with the reaction $\mathrm{C}^{+}+\mathrm{e}^{-} \rightarrow \mathrm{C}^{*}$, where $\mathrm{C}^{+}$and $\mathrm{C}^{*}$ are cationic and excited versions of the cage respectively) should be lower than the electron affinity of the analyte (EA, the potential associated with the reaction $\mathrm{A}+\mathrm{e}^{-}$ $\rightarrow \mathrm{A}^{-}$). A calculation of the excited state ionization potential of the cage modeled using cluster model $\mathbf{1}$ and the combination of TD-B3LYP and the def2-TZVP basis-set yields a value of -3.02 $\mathrm{V}$ vs the saturated calomel electrode (SCE) at the ground state geometry and $-3.22 \mathrm{~V}$ or $-3.38 \mathrm{~V}$ vs SCE at the relaxed excited state geometry, using geometry (2) or (4) respectively. These values, as can be seen in Figure 4, are both significantly lower than the predicted range of the vertical and adiabatic electron affinities of the analytes, and the excited cage should thus be able to photoreduce each of the analytes. Calculations with the DZP basis-set instead of def2-TZVP give, as can be seen in section 6 of the Supporting Information, slightly different potential values but predict overall the same relative alignment of the IP* of the cage versus the EA of the analytes. Photoinduced electron transfer thus appears a viable dynamic fluorescence quenching mechanism in the presence of all the analytes.

The Potential of Static Quenching. We now consider the likelihood of the formation of host-guest complexes with the cage for the different analytes, as well as the optical properties of such complexes and their potential role in fluorescence quenching.

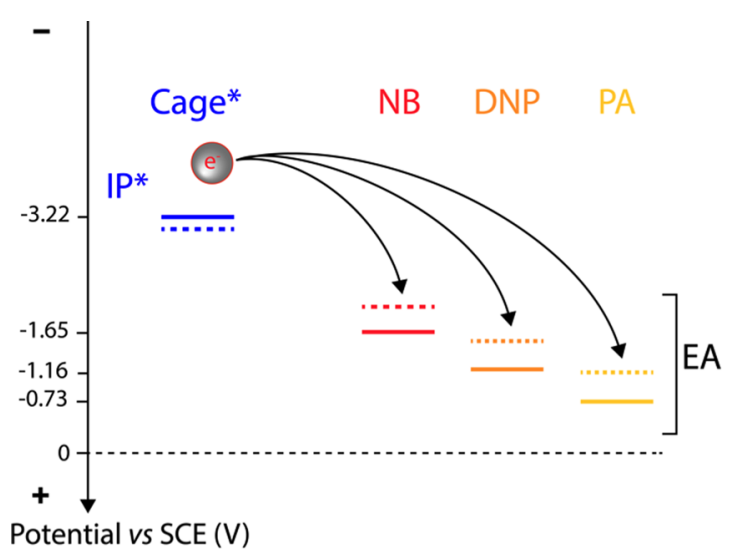

Figure 4. Excited state ionization potential (IP*) of the cage, modeled by cluster model $\mathbf{1}$, and electron affinities (EA) of the different analytes. Vertical potentials represented as dashed lines and adiabatic potentials as solid lines.

Analyte Adsorption in the Cage Host: The Role of the Secondary Amine Groups. We considered the adsorption of $\mathrm{NB}, 24 \mathrm{DNP}$ and PA in the cage host by means of docking calculations using $\mathrm{B} 3 \mathrm{LYP}+\mathrm{D} 3$ on the combination of the analyte guest and one of two cluster models of the cage; the cluster model centered around the triphenylene unit used above (1) and an alternative cluster model centered around the benzene ring (2, see Figure 5 and Scheme 3). Use of these

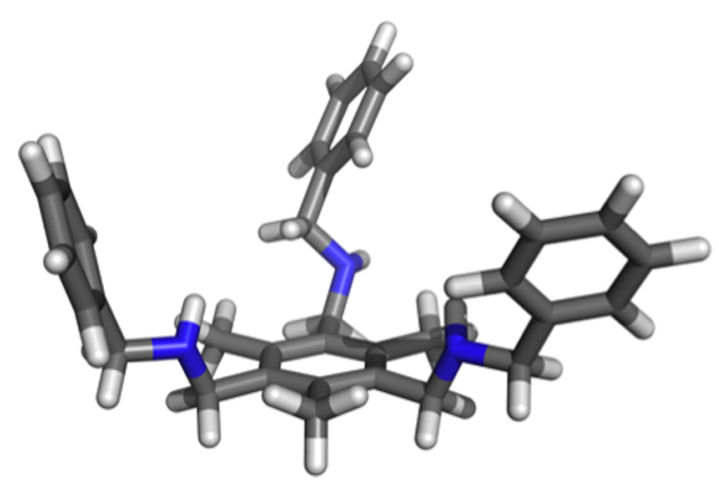

Figure 5. B3LYP/DZP optimized 3D structure of cluster model 2.

cluster models in the docking calculations rather than the whole cage will probably result in an overestimation of the calculated host-guest binding (free) energies and stability constants because the former are more flexible and less structurally constrained than the latter. However, we expect at least the predicted ordering of binding (free) energies and host-guest complex stability constants for the different analytes to be the 
Scheme 3. Structure of Cluster Model 2<smiles>Cc1ccc(CNCc2c(C)c(CNCc3ccc(C)cc3)c(C)c(CNCc3ccc(C)cc3)c2C)cc1</smiles>

same for the combination of cluster models and the whole cage and suspect that the absolute differences will be small. Most importantly, the use of the cluster models allows us to efficiently sample the different binding sites, including nonelectronic contributions to the free energy, with good quality basis-sets.

Besides the DZP basis-set used throughout the manuscript in combination with B3LYP, we also consider calculations that used the larger def2-TZVP basis-set, which should reduce the basis-set superposition error (BSSE) relative to DZP. The discussion below is based mostly upon the def2-TZVP results, but Tables 4, 5, S6, and S7 also contain the results obtained with the DZP basis-set. Furthermore, as discussed in the Methodology section, we also considered two approximations to the vibrational entropy contribution to the free energy; the standard harmonic approximation and the harmonic + rotor approximation by Grimme, ${ }^{52}$ in which the contribution of low frequency vibrational modes is described in terms of rigid rotors rather than harmonic oscillators.

Considering first the adsorption of neutral analytes in the cage (see Figure 6A for an example for PA and cluster model 1), the host-guest binding (free) energies in Tables 4 and 5 (harmonic + rotor approximation) and S6 and S7 (standard harmonic approximation) show that formation of a host-guest complex is predicted in all these cases to be exothermic but endergonic. Irrespective of the approximation used to calculate the free energy, the nonelectronic contributions to the binding free energy (entropy, zero-point energy etc.) are large and opposite in sign to the electronic binding energy. As a result, the room temperature stability constants for all analytes are generally predicted to be significantly smaller than one. The acid analytes 24DNP and PA, however, can also protonate the secondary and tertiary amines in the cage, forming protontransferred complexes with an ionic contribution to the hostguest interaction. Concentrating on the case of PA, it is evident from the data in Tables 4 and S6 that protonating the tertiary amine leads to a host-guest complex (PT-3) that is considerably less stable than that for neutral PA. However, protonating the secondary amine of the cage is predicted be exergonic and to lead to the formation of a proton transferred complex (PT-2, see Figure 6B) with a host-guest stability constant that is considerably larger than one and many orders of magnitude larger than that for adsorption of neutral PA.

Similarly, for 24DNP, protonation of the secondary amine in the cluster model of the host, with consequent deprotonation of the 24DNP, is predicted to result in a much more stable complex than the neutral case, with the formation of the proton-transferred complex being consistently mildly exergonic. The stabilization in the case of 24DNP relative to the neutral guest case is smaller than that for PA, which is to be expected, as PA is a stronger acid than 24DNP. Overall, based on the predicted stability constants for both adsorption sites, even if these are slightly overestimated with respect those in the actual cage because of the less constrained nature of the cluster models, the concentration of the analyte-cage host-guest complex relative to free analyte is thus expected to be larger for PA than for 24DNP and many orders of magnitude more than that in the case of NB. Moreover, while the different combination of approximations (DZP vs def2-TZVP, harmonic vs harmonic + rotor) yield slightly different absolute values, all methods predict a similar relative ordering of (binding) free energies and stability constant values for the different analytes.

The secondary amine groups in the cage thus allow the cage to act as a base and deprotonate the acid analytes (e.g., PA and 24DNP). This not only results in the formation of more stable host-guest complexes, but also the generation of the conjugate base anion of the analyte. The latter is strongly endergonic in the absence of the adsorption of the analyte in the cage (see Supporting Information, section 8). In other words, the cage is not a strong enough base to deprotonate for example, PA, without the added stabilization of the electrostatic interaction between the deprotonated analyte and protonated cage. Some of the free conjugate base anions in solution, formed for example by dissociation by trace water, however, can adsorb in the cage though the binding (free) energies and stability constants are predicted to be worse than those of the neutral case and at equilibrium most free conjugate base anion would be not adsorbed. The generally more negative binding energies

Table 4. B3LYP+D3 Predicted Host-Guest Binding Energies, Binding Free Energies Calculated within the Harmonic + Rotor Approximation and Stability Constants for Complexes between the Different Analytes and the Cage Modeled by the Cluster Model $1^{a}$

$\begin{array}{llllc}\text { guest } & \text { type } & \Delta E_{\text {bind }} & \Delta G_{\text {bind,298 }} & K_{\text {bind,298 }} \\ \text { NB } & \mathrm{N} & -0.69 /-0.49 & 0.02 / 0.23 & 5 \times 10^{-1} / 1 \times 10^{-4} \\ 24 \mathrm{DNP} & \mathrm{N} & -0.93 /-0.60 & -0.16 / 0.18 & 4 \times 10^{2} / 8 \times 10^{-4} \\ & \mathrm{PT}-2 & -1.37 /-0.95 & -0.54 /-0.12 & 1 \times 10^{9} / 1 \times 10^{2} \\ \mathrm{PA} & \mathrm{N} & -1.08 /-0.59 & -0.26 / 0.22 & 3 \times 10^{4} / 2 \times 10^{-4} \\ & \text { PT-3 } & -0.22 /-0,21 & - & - \\ & \text { PT-2 } & -1.72 /-1.28 & -0.86 /-0.42 & 3 \times 10^{14} / 1 \times 10^{7}\end{array}$

${ }^{a}$ All values are in eV, DZP and def2-TZVP results are given before and after the slash respectively, and all calculations used the COSMO dielectric screening solvation model $\left(\varepsilon_{\mathrm{r}} 9, \mathrm{DCM}\right)$. For binding free energies and stability constants calculated within the standard harmonic approximation see Table S6. 
Table 5. B3LYP+D3 Predicted Host-Guest Binding Energies, Binding Free Energies Calculated within the Harmonic + Rotor Approximation and Stability Constants for Complexes between the Different Analytes and the Cage Modeled by Cluster Model $2^{a}$

$\begin{array}{clccc}\text { guest } & \text { type } & \Delta E_{\text {bind }} & \Delta G_{\text {bind,298 }} & K_{\text {bind,298 }} \\ \text { NB } & \mathrm{N} & -0.87 /-0.60 & -0.17 / 0.10 & 8 \times 10^{2} / 2 \times 10^{-2} \\ \text { 24DNP } & \mathrm{N} & -0.75 /-0.49 & -0.03 / 0.23 & 3 / 1 \times 10^{-4} \\ & \text { PT-2 } & -1.20 /-0.91 & -0.45 /-0.16 & 4 \times 10^{7} / 5 \times 10^{2} \\ \text { PA } & \mathrm{N} & -1.21 /-0.79 & -0.47 /-0.05 & 8 \times 10^{7} / 6 \\ & \text { PT-2 } & -1.82 /-1.41 & -1.03 /-0.63 & 3 \times 10^{17} / 4 \times 10^{10}\end{array}$

${ }^{a}$ All values in eV, DZP and def2-TZVP results are given before and after the slash respectively, and all calculations used the COSMO dielectric screening solvation model $\left(\varepsilon_{\mathrm{r}} 9, \mathrm{DCM}\right)$. For binding free energies and stability constants calculated within the standard harmonic approximation see Table S7.

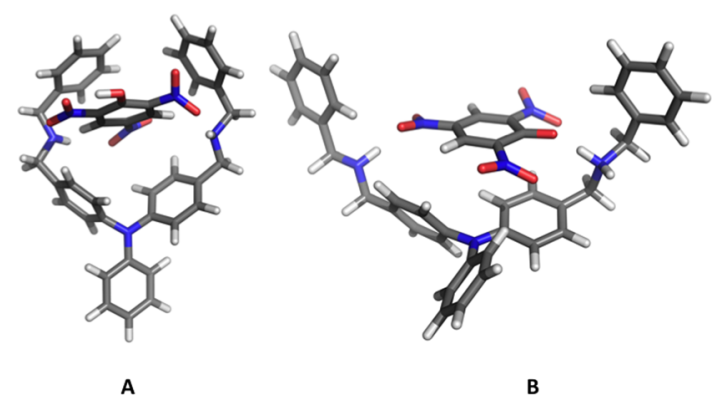

Figure 6. B3LYP/DZP optimized 3D structures of the neutral (A) and PT-2 proton transfer (B) picric acid-cluster model 1 host-guest complex.

and larger host-guest stability constants for cluster model $\mathbf{2}$ probably arise from the fact that the three secondary amine groups are located around the central benzene ring such that the explosive guest can be both close to the benzene ring for $\pi-\pi$ interactions, as well as form short contacts between $\mathrm{NO}_{2} /$ $\mathrm{O}(\mathrm{H})$ groups and amine hydrogen atoms. Finally, while we did not explicitly model the case of the Mukherjee decorated cage where the secondary amines have been converted into tertiary amines, we are confident, based upon the above data, that in this cage the acid analytes will not be deprotonated and that the differences in stability constants and analyte-cage host-guest complex concentrations for the different analytes will be a lot smaller.

Energy and Electron Transfer in the Host-Guest Complex. TD-DFT calculations on the ground state geometries of the different host-guest complexes between the analytes and the cage discussed above were then performed, using CAM-B3LYP rather than B3LYP because of the potential for CT-states in these systems. In all cases these calculations show that the lowest singlet excited state $\left(S_{1}\right)$ is predicted to be a state in which the excited electron resides on the analyte, while the hole is located on the cage (see Figure 7). Such a CT-state is how one would expect a photoinduced charge transfer to appear in a host-guest supramolecular complex. Excited states that are predominantly localized on the analyte guest, the natural end point of energy rather than electron transfer in a host-guest complex, lie higher in energy than the CT-state(s) by $\sim 0.1-0.4$ $\mathrm{eV}$ (see Table S10 in the Supporting Information). Finally, excited states that predominantly involve the cage host (the type of states that are initially excited by absorption of light), lie even higher in energy. The energetic ordering of excited states predominantly involving the analyte and the host is in line with what one would expect from the results of TD-(CAM)-B3LYP calculation on isolated molecules, presented in Tables 2 and S4.

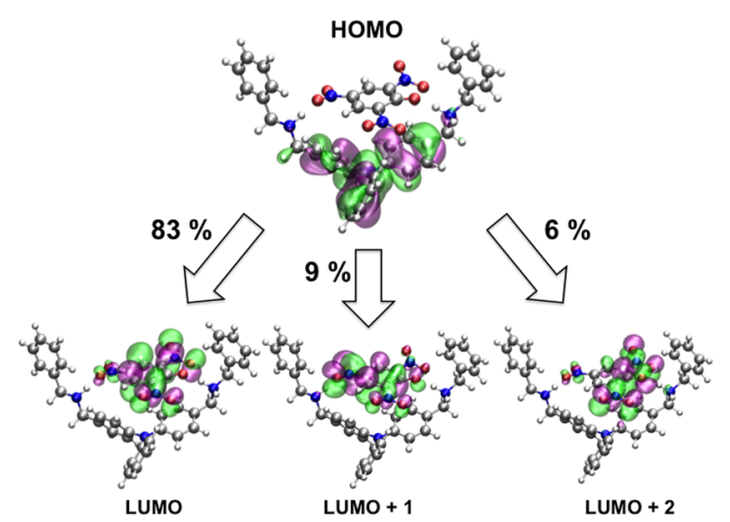

Figure 7. Overview of the major one-electron Kohn-Sham orbital excitations (e.g., HOMO $\rightarrow$ LUMO) contributing to the lowest energy TD-CAM-B3LYP singlet excited state for the PT2 proton transferred picric acid-cluster model 1 host-guest complex, clearly showing the charge-transfer character of the excited state. The percentage values besides the arrows indicate the contribution of a specific one-electron Kohn-Sham orbital excitation to the excited state (contribution with a magnitude of less than 5\% not shown). The use of green and purple signifies the different phases of the relevant orbitals; orbital plots were generated with a contour value of 0.02 .

Calculations on cluster model $\mathbf{1}$ with an adsorbed picrate anion give results that lie extremely close to those of the PT-2 PA cluster model 1 host-guest complex. Again the lowest excited state is a CT-state with the hole located on the cage and the excited electron on the picrate. This suggests, besides that the effect of the transferred proton on the photophysics itself is very small, that the origin of the conjugate base anion, deprotonated after adsorption on the cage or, before adsorption, by water in solution, has no influence on the photophysical mechanism of static quenching.

Perspective. We have found that quenching of the fluorescence of the amine molecular cage by explosive analytes must be the result of photoinduced electron transfer, be it through collision or after host-guest complex formation. Energy transfer, while often invoked in the form of FRET to explain fluorescence quenching, is predicted for the combination of amine molecular cages and nitroaromatic explosives to only be feasible for the conjugate base anion of acid explosives. Moreover, in the absence of water or other solvents that promote dissociation, it is expected that the concentration of free conjugate base anion of an acid explosive is always orders of magnitude lower than the concentration of the explosive bound in a host-guest complex by the cage.

For PA and 24DNP, our calculations predict that effectively all the explosive molecules will be present in the form of a 
host-guest complex $\left(K_{\text {bind,298 }} \gg 1\right)$, which supports the experimental observation of static quenching for the PA/ 24DNP-secondary amine cage system. For the other explosive considered, NB, our calculations predict that the majority of the explosive will be present in the free form and the case for static versus dynamic quenching is less clear based on the stability constants and the percentage of explosives complexed alone. However, when taking into account that the dynamic SternVolmer quenching constant is the product of the quenching rate constant and the inherent fluorescence lifetime of the cage and the fact that the latter is very small ( $\sim 10 \mathrm{~ns}$ experimentally, 50-170 ns from static TD-DFT calculations, see above), the lack of dynamic quenching for NB might be related to this short fluorescence lifetime. Another contributing factor might be that NB has the smallest electron affinity of all explosives and thus the smallest driving force for photoinduced electron transfer, which, in the framework of Marcus theory, suggests that it might also have a relatively small rate constant for electron transfer.

Assuming, following Kasha, rapid energy transfer from higher excited states to the lowest excited state, the photophysical mechanism of static quenching in the host-guest complex is predicted in all cases to be due to electron transfer rather than energy transfer; i.e. the lowest excited state of the analytesensor host-guest complex has charge-transfer character. Free conjugate base anions of the acid explosives, e.g., picrate, can quench the cage fluorescence through FRET/DET. However, when adsorbed, PET dominates because the attractive electrostatic interaction between electron and hole stabilizes charge-transfer states relative to local excited states. Hence in contrast to Acharyya and Mukherjee, we do not believe that FRET/DET at close proximity explains the "super-quenching ability of PA" for the amine cage.

Overall, it appears to us that the selectivity of the cage with secondary amine groups is not due to any unique photophysical properties, but rather the fact that the cage forms the most stable host-guest complexes with PA. Specifically, the predicted stability constants increase in the same order ( $\left.K_{\text {bind,298, NB }}<K_{\text {bind,298, 24DNP }}<K_{\text {bind,298, PA }}\right)$ as the experimental fluorescence quenching efficiency. Further evidence for the crucial role of the host-guest stability constant and its large size comes from the nonlinearity observed in the SternVolmer plot for PA and the amine cage, which is expected for large host-guest stability constants, as well as the experimental observation of a new feature in the absorption spectrum of the amine cage at $\sim 420 \mathrm{~nm}$ after addition of PA. The latter we expect based on our calculations to be the lowest energy excitation that is predominantly localized on the picrate anion, as the overall lowest energy CT-state is expected to have a rather weak intensity. The only minor complication with the $\sim 420 \mathrm{~nm}$ absorption feature is that, as discussed above and in the Supporting Information, the same feature might arise from PA dissociation by trace water.

The explanation in terms of analyte-sensor host-guest stability constants is also consistent with the change in selectivity for PA observed experimentally after converting the secondary amine groups into tertiary amine groups. While we have not explicitly calculated the host-guest binding free energies for the decorated cage, our work suggests that removing the secondary amine groups eliminates the ability to form PT-2 complexes and that the neutral host-guest complexes for the different explosives all have very similar binding free energies and stability constants. Indeed, the experimentally measured Stern-Volmer constant for PA and the decorated cage $\left(3.1 \times 10^{4} \mathrm{M}^{-1}\right)^{26}$ is lower than that for the cage with secondary amine groups $\left(2.2 \times 10^{5} \mathrm{M}^{-1}\right),{ }^{25}$ although one should be careful not to overinterpret these specific experimental numbers. The two experiments use different solvents (tetrahydrofuran vs dichloromethane) and one can argue about the suitability of the model used to extract the constants. Still in the case of the decorated cage, it thus appears that it is not more selective for other nitroaromatics, but instead less selective for PA.

Moving our attention to explosive sensing in general, our calculations suggest that static quenching via the formation of stable complexes between explosives and an otherwise fluorescent sensor (macro)molecule is a powerful approach. Because the strength of quenching appears to be directly determined by the strength of the complex formed and the fraction of explosive bound, it is easy to conceptualize how to improve the selectivity for a given explosive by increasing the explosive-sensor complex binding strength through careful binding site design. In the case of phenol-based molecules (PA, $24 \mathrm{DNP})$, this involves the presence of strategically located amine groups or related Lewis bases. For example, the selectivity of metal-organic frameworks based on amine group containing ligands ${ }^{12,58}$ as fluorescence quenching sensors for PA might also involve the formation of such explosivesensor host-guest complexes. However, for other explosives this might involve different functional groups or a different size or shape of binding pocket.

The second obvious combination of requirements for static quenching through PET is that the sensing (macro)molecule is electron rich, such that it has a shallow excited-state ionization potential and thus a large driving force for PET, while at the same time highly fluorescent in the absence of a quencher. Systems based around triphenylamine units obviously fulfill this requirement, but the same should hold for (macro)molecules based around many other conjugated electron-rich fragments, especially if bulk side groups prevent dimerization of such fragments and thus self-quenching through $\mathrm{H}$-aggregate formation. Indeed a recent review, ${ }^{18}$ discusses a wide range of small molecules that act as explosive sensors via fluorescence quenching that combine a conjugated electron-rich fragment with bulky side groups. Similarly, polymers that act as efficient fluorescent quenching sensors for explosives are based on electron-rich fragments that do not pack efficiently and hence minimize self-quenching. ${ }^{1,4}$

Finally, fluorescence-quenching sensors for explosives based on energy transfer are probably generally harder to achieve than those based on electron transfer (i.e., FRET/DET rather than PET), at least for sensing nitroaromatics. The fact that absorption onset values of all nitroaromatics, phenol based (e.g., PA) or not (e.g., TNT), lie close to those of many potential conjugated fluorescent fragments, means that sensor molecules based on such fragments should display only a very limited Stokes shift to be able to transfer energy via FRET/ DET to such nitroaromatics. A likely exception to this scenario is the case of the phenol based acid explosives. In these systems, as discussed above, deprotonation and conjugate base anion formation results in a sufficient red-shift of the absorption onset to make FRET/DET a more realistic option by significantly increasing the maximum amount of Stokes shift a sensor molecule can undergo and still excite those deprotonated analytes. As a result, we think the exact solvent system used in fluorescence quenching experiments can have a critical 
influence on the extent and mechanism of quenching, especially when sufficiently polar or basic to form significant amounts of conjugate base anions in solution, and needs to be carefully considered. The fluorescence quenching mechanism in dry organic solvents is likely to be different than that in water.

\section{CONCLUSIONS}

In conclusion, we show that fluorescence quenching of amine molecular cages by nitroaromatic explosives in nonpolar solvents is the result of the formation of a stable supramolecular complex with a lowest excited state with charge-transfer character. We demonstrate that the high sensitivity and specificity for picric acid of such an amine molecular cage with secondary amine groups is the result of the formation of a very stable proton-transferred complex, involving protonation of the secondary amine and formation of an adsorbed picrate anion, and is thus linked to the chemistry and geometry of the adsorption site. Moreover, we discuss how trace water could be responsible for picrate anion formation in this and other systems. Finally, we analyze the more general requirements for explosive sensors based upon static fluorescence quenching and discuss that sensors based on dynamic fluorescence quenching by energy transfer are difficult to achieve other than for acid explosives in very polar solvents because of constraints on the maximum allowed Stokes shift of the sensor.

\section{ASSOCIATED CONTENT}

\section{S Supporting Information}

The Supporting Information is available free of charge on the ACS Publications website at DOI: 10.1021/acs.jpcb.6b03059.

Further information on the different conformations of the amine cage, the vertical excitation spectra of cluster model $\mathbf{1}$, the geometry change between the ground state and excited state minimum energy geometry, the calculated redox potentials, and the free energy calculations, as well as $x y z$ coordinates of the DFT optimized structure (PDF)

\section{AUTHOR INFORMATION}

\section{Corresponding Author}

*(M.A.Z.) E-mail: m.zwijnenburg@ucl.ac.uk.

\section{Notes}

The authors declare no competing financial interest.

\section{ACKNOWLEDGMENTS}

We kindly acknowledge Dr. Jan Gerit Brandenburg, Ms. Monika Jurcic and Dr. Aaron Thornton for stimulating discussions. M.A.Z. and K.E.J. acknowledge the U.K. Engineering and Physical Sciences Research Council (EPSRC) for a Career Acceleration Fellowship (Grant EP/I004424/1) and the Royal Society for a University Research Fellowship, respectively. K.E.J. further acknowledges the EPSRC for additional funding (EP/M017257/1). W.J.P. is grateful for an EPSRC Doctoral Prize Fellowship (EP/M506448/1). Finally, computational time on ARCHER, the U.K.'s national high-performance computing service (via our membership of the UK's HPC Materials Chemistry Consortium, which is funded by EPSRC Grant EP/L000202/1), and the EPSRC National Service for Computational Chemistry Software is gratefully acknowledged.

\section{REFERENCES}

(1) Yang, J. S.; Swager, T. M. Porous Shape Persistent Fluorescent Polymer Films: An Approach to TNT Sensory Materials. J. Am. Chem. Soc. 1998, 120, 5321-5322.

(2) Yang, J. S.; Swager, T. M. Fluorescent Porous Polymer Films as Tnt Chemosensors: Electronic and Structural Effects. J. Am. Chem. Soc. 1998, 120, 11864-11873.

(3) Toal, S. J.; Trogler, W. C. Polymer Sensors for Nitroaromatic Explosives Detection. J. Mater. Chem. 2006, 16, 2871-2883.

(4) Thomas, S. W.; Joly, G. D.; Swager, T. M. Chemical Sensors Based on Amplifying Fluorescent Conjugated Polymers. Chem. Rev. 2007, 107, 1339-1386.

(5) Lan, A. J.; Li, K. H.; Wu, H. H.; Olson, D. H.; Emge, T. J.; Ki, W.; Hong, M. C.; Li, J. A Luminescent Microporous Metal-Organic Framework for the Fast and Reversible Detection of High Explosives. Angew. Chem., Int. Ed. 2009, 48, 2334-2338.

(6) Gole, B.; Bar, A. K.; Mukherjee, P. S. Fluorescent Metal-Organic Framework for Selective Sensing of Nitroaromatic Explosives. Chem. Commun. 2011, 47, 12137-12139.

(7) Zhang, C. Y.; Che, Y. K.; Zhang, Z. X.; Yang, X. M.; Zang, L. Fluorescent Nanoscale Zinc(II)-Carboxylate Coordination Polymers for Explosive Sensing. Chem. Commun. 2011, 47, 2336-2338.

(8) Pramanik, S.; Zheng, C.; Zhang, X.; Emge, T. J.; Li, J. New Microporous Metal-Organic Framework Demonstrating Unique Selectivity for Detection of High Explosives and Aromatic Compounds. J. Am. Chem. Soc. 2011, 133, 4153-4155.

(9) Nagarkar, S. S.; Joarder, B.; Chaudhari, A. K.; Mukherjee, S.; Ghosh, S. K. Highly Selective Detection of Nitro Explosives by a Luminescent Metal-Organic Framework. Angew. Chem., Int. Ed. 2013, $52,2881-2885$

(10) Hu, Z. C.; Deibert, B. J.; Li, J. Luminescent Metal-Organic Frameworks for Chemical Sensing and Explosive Detection. Chem. Soc. Rev. 2014, 43, 5815-5840.

(11) Jurcic, M.; Peveler, W. J.; Savory, C. N.; Scanlon, D. O.; Kenyon, A. J.; Parkin, I. P. The Vapour Phase Detection of Explosive Markers and Derivatives Using Two Fluorescent Metal-Organic Frameworks. J. Mater. Chem. A 2015, 3, 6351-6359.

(12) Nagarkar, S. S.; Desai, A. V.; Ghosh, S. K. Engineering MetalOrganic Frameworks for Aqueous Phase 2,4,6-Trinitrophenol (TNP) Sensing. CrystEngComm 2016, 18, 2994-3007.

(13) Bar, A. K.; Shanmugaraju, S.; Chi, K. W.; Mukherjee, P. S. SelfAssembly of Neutral and Cationic Pd-Ii Organometallic Molecular Rectangles: Synthesis, Characterization and Nitroaromatic Sensing. Dalton Trans. 2011, 40, 2257-2267.

(14) Freeman, R.; Willner, I. Optical Molecular Sensing with Semiconductor Quantum Dots (Qds). Chem. Soc. Rev. 2012, 41, 4067-4085.

(15) Freeman, R.; Finder, T.; Bahshi, L.; Gill, R.; Willner, I. Functionalized CdSe/ZnS Qds for the Detection of Nitroaromatic or RDX Explosives. Adv. Mater. 2012, 24, 6416-6421.

(16) Enkin, N.; Sharon, E.; Golub, E.; Willner, I. Ag Nanocluster/ DNA Hybrids: Functional Modules for the Detection of Nitroaromatic and RDX Explosives. Nano Lett. 2014, 14, 4918-4922.

(17) Peveler, W. J.; Roldan, A.; Hollingsworth, N.; Porter, M. J.; Parkin, I. P. Multichannel Detection and Differentiation of Explosives with a Quantum Dot Array. ACS Nano 2016, 10, 1139-1146.

(18) Shanmugaraju, S.; Mukherjee, P. S. Pi-Electron Rich Small Molecule Sensors for the Recognition of Nitroaromatics. Chem. Commun. 2015, 51, 16014-16032.

(19) Mukherjee, S.; Desai, A. V.; Inamdar, A. I.; Manna, B.; Ghosh, S. K. Selective Detection of 2,4,6-Trinitrophenol (TNP) by a Pi-Stacked Organic Crystalline Solid in Water. Cryst. Growth Des. 2015, 15, 3493-3497.

(20) Francesconi, O.; Ienco, A.; Moneti, G.; Nativi, C.; Roelens, S. A Self-Assembled Pyrrolic Cage Receptor Specifically Recognizes BetaGlucopyranosides. Angew. Chem., Int. Ed. 2006, 45, 6693-6696.

(21) Liu, X. J.; Liu, Y.; Li, G.; Warmuth, R. One-Pot, 18-Component Synthesis of an Octahedral Nanocontainer Molecule. Angew. Chem., Int. Ed. 2006, 45, 901-904. 
(22) Mastalerz, M. One-Pot Synthesis of a Shape-Persistent EndoFunctionalised Nano-Sized Adamantoid Compound. Chem. Commun. 2008, 4756-4758.

(23) Tozawa, T.; Jones, J. T. A.; Swamy, S. I.; Jiang, S.; Adams, D. J.; Shakespeare, S.; Clowes, R.; Bradshaw, D.; Hasell, T.; Chong, S. Y.; et al. Porous Organic Cages. Nat. Mater. 2009, 8, 973-978.

(24) Rowan, S. J.; Cantrill, S. J.; Cousins, G. R. L.; Sanders, J. K. M.; Stoddart, J. F. Dynamic Covalent Chemistry. Angew. Chem., Int. Ed. 2002, 41, 898-952.

(25) Acharyya, K.; Mukherjee, P. S. A Fluorescent Organic Cage for Picric Acid Detection. Chem. Commun. 2014, 50, 15788-15791.

(26) Acharyya, K.; Mukherjee, P. S. Postsynthetic Exterior Decoration of an Organic Cage by Copper(I)-Catalysed a(3)Coupling and Detection of Nitroaromatics. Chem. - Eur. J. 2015, 21, 6823-6831.

(27) Brutschy, M.; Schneider, M. W.; Mastalerz, M.; Waldvogel, S. R. Porous Organic Cage Compounds as Highly Potent Affinity Materials for Sensing by Quartz Crystal Microbalances. Adv. Mater. 2012, 24, 6049-6052.

(28) Lee, T. C.; Kalenius, E.; Lazar, A. I.; Assaf, K. I.; Kuhnert, N.; Grun, C. H.; Janis, J.; Scherman, O. A.; Nau, W. M. Chemistry inside Molecular Containers in the Gas Phase. Nat. Chem. 2013, 5, 376-382.

(29) Mitra, T.; Jelfs, K. E.; Schmidtmann, M.; Ahmed, A.; Chong, S. Y.; Adams, D. J.; Cooper, A. I. Molecular Shape Sorting Using Molecular Organic Cages. Nat. Chem. 2013, 5, 276-281.

(30) Chen, L.; Reiss, P. S.; Chong, S. Y.; Holden, D.; Jelfs, K. E.; Hasell, T.; Little, M. A.; Kewley, A.; Briggs, M. E.; Stephenson, A.; et al. Separation of Rare Gases and Chiral Molecules by Selective Binding in Porous Organic Cages. Nat. Mater. 2014, 13, 954-960.

(31) Kewley, A.; Stephenson, A.; Chen, L. J.; Briggs, M. E.; Hasell, T.; Cooper, A. I. Porous Organic Cages for Gas Chromatography Separations. Chem. Mater. 2015, 27, 3207-3210.

(32) Hasell, T.; Miklitz, M.; Stephenson, A.; Little, M. A.; Chong, S. Y.; Clowes, R.; Chen, L. J.; Holden, D.; Tribello, G. A.; Jelfs, K. E.; et al. Porous Organic Cages for Sulfur Hexafluoride Separation. J. Am. Chem. Soc. 2016, 138, 1653-1659.

(33) Becke, A. D. Density-Functional Thermochemistry 0.3. The Role of Exact Exchange. J. Chem. Phys. 1993, 98, 5648-5652.

(34) Becke, A. D. Density-Functional Exchange-Energy Approximation with Correct Asymptotic-Behavior. Phys. Rev. A: At., Mol., Opt. Phys. 1988, 38, 3098-3100.

(35) Grimme, S.; Antony, J.; Ehrlich, S.; Krieg, H. A Consistent and Accurate Ab Initio Parametrization of Density Functional Dispersion Correction (DFT-D) for the 94 Elements H-Pu. J. Chem. Phys. 2010, 132, 154104.

(36) Yanai, T.; Tew, D. P.; Handy, N. C. A New Hybrid ExchangeCorrelation Functional Using the Coulomb-Attenuating Method (CAM-B3LYP). Chem. Phys. Lett. 2004, 393, 51-57.

(37) Christiansen, O.; Koch, H.; Jorgensen, P. The 2nd-Order Approximate Coupled-Cluster Singles and Doubles Model CC2. Chem. Phys. Lett. 1995, 243, 409-418.

(38) Hattig, C.; Weigend, F. CC2 Excitation Energy Calculations on Large Molecules Using the Resolution of the Identity Approximation. J. Chem. Phys. 2000, 113, 5154-5161.

(39) Peach, M. J. G.; Benfield, P.; Helgaker, T.; Tozer, D. J. Excitation Energies in Density Functional Theory: An Evaluation and a Diagnostic Test. J. Chem. Phys. 2008, 128, 044118.

(40) Ahlrichs, R.; Bar, M.; Haser, M.; Horn, H.; Kolmel, C. Electronic-Structure Calculations on Workstation Computers - the Program System Turbomole. Chem. Phys. Lett. 1989, 162, 165-169.

(41) Furche, F.; Ahlrichs, R.; Haettig, C.; Klopper, W.; Sierka, M.; Weigend, F. Turbomole. WIREs Comput. Mol. Sci. 2014, 4, 91-100.

(42) Schmidt, M. W.; Baldridge, K. K.; Boatz, J. A.; Elbert, S. T.; Gordon, M. S.; Jensen, J. H.; Koseki, S.; Matsunaga, N.; Nguyen, K. A.; $\mathrm{Su}, \mathrm{S}$. J.; et al. General Atomic and Molecular Electronic-Structure System. J. Comput. Chem. 1993, 14, 1347-1363.

(43) Valiev, M.; Bylaska, E. J.; Govind, N.; Kowalski, K.; Straatsma, T. P.; Van Dam, H. J. J.; Wang, D.; Nieplocha, J.; Apra, E.; Windus, T. L.; et al. NWchem: A Comprehensive and Scalable Open-Source
Solution for Large Scale Molecular Simulations. Comput. Phys. Commun. 2010, 181, 1477-1489.

(44) Schafer, A.; Horn, H.; Ahlrichs, R. Fully Optimized Contracted Gaussian-Basis Sets for Atoms Li to Kr. J. Chem. Phys. 1992, 97, 25712577.

(45) Hehre, W. J.; Ditchfie, R.; Pople, J. A. Self-Consistent Molecular-Orbital Methods 0.12. Further Extensions of GaussianType Basis Sets for Use in Molecular-Orbital Studies of OrganicMolecules. J. Chem. Phys. 1972, 56, 2257-2261.

(46) Hariharan, P. C.; Pople, J. A. Influence of Polarization Functions on Molecular-Orbital Hydrogenation Energies. Theor. Chim. Acta 1973, 28, 213-222.

(47) Weigend, F.; Ahlrichs, R. Balanced Basis Sets of Split Valence, Triple Zeta Valence and Quadruple Zeta Valence Quality for H to Rn: Design and Assessment of Accuracy. Phys. Chem. Chem. Phys. 2005, 7, 3297-3305.

(48) Klamt, A.; Schuurmann, G. Cosmo: A New Approach to Dielectric Screening in Solvents with Explicit Expressions for the Screening Energy and Its Gradient. J. Chem. Soc., Perkin Trans. 2 1993, 799-805.

(49) Miertus, S.; Scrocco, E.; Tomasi, J. Electrostatic Interaction of a Solute with a Continuum - a Direct Utilization of Abinitio Molecular Potentials for the Prevision of Solvent Effects. Chem. Phys. 1981, 55, $117-129$.

(50) Tomasi, J.; Mennucci, B.; Cammi, R. Quantum Mechanical Continuum Solvation Models. Chem. Rev. 2005, 105, 2999-3094.

(51) Marenich, A. V.; Cramer, C. J.; Truhlar, D. G. Universal Solvation Model Based on Solute Electron Density and on a Continuum Model of the Solvent Defined by the Bulk Dielectric Constant and Atomic Surface Tensions. J. Phys. Chem. B 2009, 113, $6378-6396$

(52) Grimme, S. Supramolecular Binding Thermodynamics by Dispersion-Corrected Density Functional Theory. Chem. - Eur. J. 2012, 18, 9955-9964.

(53) Santolini, V.; Tribello, G. A.; Jelfs, K. E. Predicting Solvent Effects on the Structure of Porous Organic Molecules. Chem. Commun. 2015, 51, 15542-15545.

(54) Davis, M. M.; Paabo, M. Spectrophotometric Determination of Thermodynamic Pk Value of Picric Acid in Water at 25 Degrees C. J. Res. Natl. Bur. Stand., Sect. A 1963, 67A, 241-246.

(55) Kolthoff, I. M.; Chantooni, M. K. Effect of Water on Dissociation and Dissociation Constant of Picric Acid in Acetonitrile. J. Am. Chem. Soc. 1969, 91, 6907-6910.

(56) Guiglion, P.; Butchosa, C.; Zwijnenburg, M. A. Polymeric Watersplitting Photocatalysts; a Computational Perspective on the Water Oxidation Conundrum. J. Mater. Chem. A 2014, 2, 1199612004.

(57) Guiglion, P.; Berardo, E.; Butchosa, C.; Wobbe, M. C. C.; Zwijnenburg, M. A. Modelling Materials for Solar Fuel Synthesis by Artificial Photosynthesis; Predicting the Optical, Electronic and Redox Properties of Photocatalysts. J. Phys.: Condens. Matter 2016, 28, 074001.

(58) Nagarkar, S. S.; Desai, A. V.; Samanta, P.; Ghosh, S. K. Aqueous Phase Selective Detection of 2,4,6-Trinitrophenol Using a Fluorescent Metal-Organic Framework with a Pendant Recognition Site. Dalton Trans. 2015, 44, 15175-15180. 